\title{
Urgences
}

\section{Alys en cinémascope - ou la vie est un songe}

\section{Robert Dion}

Numéro 26, décembre 1989

Des textes qui chantent

URI : https://id.erudit.org/iderudit/025556ar

DOI : https://doi.org/10.7202/025556ar

Aller au sommaire du numéro

Éditeur(s)

Urgences

ISSN

0226-9554 (imprimé)

1927-3924 (numérique)

Découvrir la revue

Citer cet article

Dion, R. (1989). Alys en cinémascope - ou la vie est un songe. Urgences, (26), 64-73. https://doi.org/10.7202/025556ar d'utilisation que vous pouvez consulter en ligne.

https://apropos.erudit.org/fr/usagers/politique-dutilisation/ 


\section{Alys en cinémascope 1 - ou la vie est un songe Robert Dion}

Au Mocambo

Quand tu chantais \& Tico Tico Tico *

Les nuits de Montréal

Valaient bien celles d'la place Pigalle

5 Les chas chas, les mambos...

Et les hommes étaient beaux

En c'temps-ia

Souliers blancs et panamas...

Quand ils entraient cigare au bec

10 [I] crachaient leurs dollars

Pour écouter chanter une star

La première star du Québec

Et quand vers minuit

S'avançait le M.C.

15 Il disait: $\propto$ [Mesdames, messieurs] ${ }^{2}$ La voici

Alys Robi »

Tu paraissais

Et tu chantais "Tico Tico Tico"

Tu faisais tout ton show

20

Portée par les bravos

Le maire et la mafia

Tout l'monde étaít là

A tes pieds

C'était comme au cinéma

25 Tu voyais ta vie

Comme un film en cinémascope

C'était toi la reine des années quarante

Aux États-Unis

Tu rêvais d'arriver au top

30 Et de soir en soir

1 Paroles de Luc Plamondon, musique de Germain Gauthier; interprete: Diane Dufresne; album: Strip Tease, Barclay, 1979 - Barclay 80294.

2 Les crochets signalent les differences entre la version imprimée au dos de la pochette et la version chantée. Nous n'avons cependant pas supprimé les $\theta$ élidés par la chanteuse; il nous a semblé préférable de respecter le plus possible la transcription voulue par Plamondon. 
Tu poursuivais ta gloire Comme une étoile filante

Hôtels de luxe, avion privé

Et lumières de Broadway

35 Oui, c'était toi « Alys

Au pays des merveilles »

Mais derrière le miroir

On ne trouve pas toujours le soleil

Un beau jour, tout a craqué

40 Tu vivais ta vie

Comme un film en cinémascope

[T'étais] la reine des années quarante

Comme n'importe qui

Tas eu des hits [et pis] des flops

45 Mais les années noires

Sont loin dans ta mémoire

Aujourd'hui quand tu chantes

Quand tu chantes dans les clubs d'la Main

C'est encore toi la reine

50 Tous tes vieux fans sont là

Pour te tendre les bras

Et ton avion privé

Tattend pour t'emmener à Broadway

« Somewhere over the rainbow... *

55 [C'était toi la reine des années quarante]

[Oh] Y'a sûrement des jours

Où tu voudrais pouvoir dire stop

Mais chanter

C'est ta façon

60 De rester vivante

Quand on voit sa vie

Comme un film en cinémascope

Parfois on fait des hits

Parfois on fait des flops

65 [...] Au moins on se dit

$J$ 'ai voulu arriver au top

Après tout tant pis

Si on n'sait pas dire stop

Quand on voit sa vie

70 En cinémascope

Quand on voit sa vie

En cinémascope 
66

Parmi les nombreux textes que Luc Plamondon a écrits pour Diane Dufresne, Alys en cinémascope est sans doute l'un des plus représentatifs. S'y déploient, en effet, quelques topics privilégiés, dont l'emprise est telle qu'on peut en interroger l'origine: Plamondon ou Dufresne? Il est difficile, ici, d'effectuer le partage habituel entre auteur et interprète, tant les deux univers, tels qu'on les connait par ailleurs, semblent coïncider.

La collaboration entre Dufresne et Plamondon est particulière, dans la mesure où Dufresne s'avère, peu ou prou, la coauteure du texte. Son personnage n'a pas été créé par l'auteur, mais seulement transcrit. On ne manquera pas, au vu de certains indices textuels qui donnent à croire que Diane Dufresne entend, malgré le $t u$ allocutif d'Alys en cinémascope, s'approprier l'histoire racontée ${ }^{3}$ (en l'occurrence, celle d'Alys Robi ${ }^{4}$ ), de noter l'insertion de ce texte dans ce qu'il faut bien nommer une mythologie dufresnienne, largement publicisée. Mais, dans l'ignorance où nous sommes de la part biographique (Dufresne) ou littéraire (Plamondon) de cette mythologie, mieux vaut considérer Alys en cinémascope comme le produit d'un dispositif énonciatif essentiellement ambigu, sinon volontairement brouillé. Notre analyse se placera donc sous le signe de la méfiance.

Dans Alys en cinémascope, nous alerte, d'entrée de jeu, la graphie inhabituelle du prénom. Sans trop accorder d'importance à ce qui constitue sans doute un hasard, notons que tout se passe comme si, par une prescience cratylienne, le prénom même de la star "déchue ", Alys, appelait "abysse ", c'est-àdire «abîme . La transgression de l'orthographé habituelle Alice - semble appeler le châtiment, la chute dans l'“ abysse *. Rappelons que cette chute dans l'abysse sera matérialisée par

3 Ne citons ici que le conditionnel de la huitième strophe, qui permet à l'interprète-narratrice de se a mettre à la place " du personnage d'Alys Robi.

4 Alice Robitaille est née le 3 février 1923, dans le quartier Saint-Sauveur à Québec. Très jeune, elle participe au concours des jeunes talents Catelli et entreprend une carrière professionnelle. Suit une période de travail intense, qui la mène d'abord à Montréal (où elle cótoie des artistes de vaudeville tels que Rose Ouellette et Olivier Guimond fils), puis sur les scènes américaines et europénnes. À 23 ans, brisée physiquement et moralement, elle entre en maison de repos. Elle est ensuite internee à l'hópital psychiatrique Saint-Michel-Archange pendant cinq ans. Au sortir de l'hópital, en 1951, sa fortune est dilapidée et sa carrière, réduite à néant. Elle recommence alors à chanter dans les cabarets, sans plus jamais atteindre les sommets de la popularité. 
une mutilation, puisque Alys Robi subira une lobotomie durant son séjour à l'hôpital psychiatrique. Or, il est curieux de constater que cette mutilation trouve aussi un écho dans le nom de la chanteuse: Robi provient de Robitaille, nom littéralement re-taillé, donc mutilé. D'une certaine façon, la destinée de la chanteuse semble inscrite dans son nom même. De fait, ce nom choisi confêre à la chanteuse un statut d'artiste, c'està-dire un statut symbolique particulier. Il lui garantit, au surplus, l'extra-territorialité corrélative à cette symbolique: le nom d'«Alys Robi», en effet, ne sonne ni québécois, ni anglosaxon, ni même latin; il sonne un peu comme tout cela à la fois.

Certes, il n'est pas étonnant que Plamondon ait été tenté de jouer avec le prénom de son personnage. On verra qu'entre Alys et Alice au pays des merveilles, le pas est vite franchi. Un tel prénom constitue en fait un véritable blason: comme tel, il appelle la description et suscite les variations; bref, il incite à blasonner ${ }^{5}$.

Quant au programme du texte, il est inscrit dans le titre de la chanson. Alys en cinémascope, c'est Alys vue par le truchement d'un médium irréalisant, le cinéma. Le procédé cinémascope ajoute encore à ce Verfremdungseffekt, puisque ce procédé de cinéma sur écran large implique une déformation de l'image. De même, la mythification de la destinée d'Alys Robi implique une déformation de la stricte vérité documentaire. Pour être plus large, plus englobante, l'image doit renoncer à être fidèle; elle doit se désingulariser. Ainsi, le texte d'Alys en cinémascope pose d'emblée son irréalité; il va même jusqu'à nommer le prisme qui le déforme: le cinéma. Dans un autre ordre d'idées, on pourrait même ajouter que le texte est ouvertement mythomane: nous sommes dans le domaine du "plus grand que nature», du «bigger than life». Telle est, ici, la nature de l'anamorphose textuelle.

La chanson compte trois parties. La première correspond aux deux premières strophes, la seconde, aux trois strophes

5 On pourrait multiplier à l'infini les rêveries onomastiques. Notons seulement encore la symétrie du nom et du prénom, qui comptent chacun le méme nombre de lettres, et le même nombre de consonnes et de voyelles (disposées voyelleconsonne-voyelle-consonne dans le prénom, consonne-voyelle-consonnevoyelle dans le nom). En anticipant sur la suite de notre analyse, disons qu'il s'agit de l'un de ces effets de miroir caractéristiques du texte a l'étude. Nous remercions ici André Gervais pour ses précieuses observations. 
68

suivantes, et la troisième, aux quatre dernières strophes. Cette subdivision s'appuie à la fois sur des critères formels et thématiques.

La première partie est essentiellement documentaire: la syntaxe parataxique (accumulative - v. 5-9) contribue à accélérer la description et à poser le cadre: le monde des cabarets montréalais des années quarante, tels que le Mocambo ou la Casa Loma, où officiaient vedettes canadiennes-françaises et américaines. L'authenticité de cette description est assurée par un dispositif citationnel. Plamondon évoque d'abord une chanson à succès interprétée par Alys Robi, Tico Tico (paroles françaises de Jacques Larue 6; musique de Zequinha Abreu), puis une chanson interprétée par Jacques Normand, Les nuits de Montréal (1949: paroles de Jean Rafa; musique de Mimille Prudhomme). Dans l'enregistrement, l'illusion réaliste est appuyée par la voix de Jacques Normand, qui dit les vers 15 et 16, jouant ainsi son propre rôle, celui du maître de cérémonie.

Il appert, ainsi, que ces airs à succès constituent des effets de reel. Ils pointent la culture dans laquelle se meut Alys Robi. Du reste, on pourrait ajouter que la chanson dont il est ici question représente en quelque sorte, du point de vue musical et textuel, une transposition attendrie de ces chansons des années quarante.

Est par ailleurs indexée, dans cette première partie, l'étrangeté irréductible de ce milieu des cabarets. Paradoxalement, il semble bien que la description réaliste ait pour fonction thématique de marquer une prise de distance, qui sera accentuée dans la seconde partie du texte. Quoique tangible, réel, ce milieu est celui de l'irréalité la plus radicale ${ }^{7}$ et, plus précisément, celui de l'absence d soi. On en veut pour preuve les nombreux lexèmes indexant la latinité ou l'étrangeté en général: "Mocambo * (v. 1), "Tico Tico Tico * (v. 2), «Pigalle " (v. 4), « chas chas * (v. 5), « mambos * (v. 5), « panamas» (v. 8). Ces traits sémiques ont leur importance ${ }^{8}$, quand on sait que la chanson évoque la schizophrénie (v. 35-39) et la mythomanie,

6 Les paroles anglaises sont signées Irwin Drake.

7 Ainsi, le monde du cabaret ne refiète en aucune façon la société canadiennefrançaise qu'il a pour fonction de divertir. C'est pourquoi le nationalisme professe par Alys Robi dans sa pseudo-autobiographie (Alys Robi, ma carrière et ma vie, Montréal, Éd. Québecor, 1980) fait sourire: elle se réjouit de ce qu'il se trouve, à partir des années quarante, des Québécois pour singer les Américains et les Latino-Américains.

8 On les retrouve aussi dans les autres parties du texte, ou ils sont toutefois plus rares: « mafia» (v.21), « États-Unis " (v.28), « Broadway " (v.34), etc. 
lesquelles constituent peut-être deux façons complémentaires de poser le problème de l'altérité.

La première partie du texte s'achève lorsque *la première star du Québec * ${ }^{9}$, enfin nommée, entre littéralement en scène:

II disait : $\alpha$ [Mesdames, messieurs] La voici

Alys Robi \# (v. 15-16)

Se met en place, dès lors, tout un vocabulaire de l'évanescence: tu paraissais (v. 17), portée par les bravos (v. 20), c'était comme au cinéma (v. 24). Le récit de l'apogée d'une star - qui fonde l'unité thématique de la seconde partie du texte - est celui de la circularité absolue du sens. Le monde du spectacle renvoie au cinéma comme un miroir reflète un autre miroir: tourniquet infini, cercle vicieux, Teufelskreis.

Le vingt-quatrième vers, "C'était comme au cinéma*, constitue à proprement parler le pivot du texte. Il renoue avec la thématique cinématographique annoncée par le titre, signalant ainsi la principale isotopie sémantique du discours. En outre, ce vers contribue à mettre le texte en communication avec tout un intertexte. On pense à certaines chansons de Plamondon interprétées par Dufresne, telles que Chanson pour Elvis, La chanteuse straight, Les adieux d'un sex symbol. Ces chansons ont ceci de commun qu'elles puisent au mythe de la star et qu'elles proposent le récit des grandeurs et misères du métier ${ }^{10}$ d'entertainer; elles peignent des rockeuses et des rockeurs "sanctifiés * aux prises avec les phénomènes du vieillissement, de la folie ou, plus prosaïquement, de la perte de popularité. Cette thématique est si prégnante qu'elle a façonné l'image de Dufresne, allant même jusqu'à modeler sa biographie ${ }^{11}$. C'est d'ailleurs l'adhésion profonde

9 Ce vers particulièrement maladroit prend un certain relief lorsqu'il est chan par Diane Dufresne. On ne saurait rendre compte ici de lapport que représente Pinterprétation de la chanteuse: par sa façon trés singulière d'accentuer les mots, elle corrige les irrégularités rythmiques du texte; dans sa bouche, «ain v (v. 48) rime avec a reine " (v. 49) et les vers les plus plats sont rendus avec une conviction sans défaut. 10 Mais s'agit-il encore d'un métier?

11 Les univers de Plamondon et de Dufresne convergent ici jusqu'à se confondre. On n'a jamais pu se prononcer avec certitude sur le statut de Diane Dufresne: est-elle star, diva, vedette, chanteuse? II est sor, en tout cas, que quel que soit ce statut, il a été façonné par ce type de chansons puisant aux sources de la mythologie américaine: "Mourir comm' Janis / Dans un motel cheap / Loin des spotlights, près du soleil / Loin des spotlights, près du soleil " (La chanteuse straight). 
de Diane Dufresne au mythe de la star déchue qui rend si invitante son assimilation à Alys Robi.

La quatrième strophe marque l'affirmation d'une subjectivité absolue ("Tu voyais ta vie» - v. 25) portée vers l'irréalité («Comme un film en cinémascope - - v. 26). En fait, la strophe est construite sur une alternance subtile entre irréalité et réalité. Le mouvement est celui d'un champ-contrechamp. Du phantasme ( «tu te voyais» - v. 25-26), on passe à la réalité (" tu étais la reine des années quarante" - v. 27), puis de nouveau au phantasme (" tu rêvais» - v. 29-32). Si ici l'expression " arriver au top * (v. 29) ne rime pas encore avec « stop * (v. 66 et $68)$, elle semble déjà appeler une semblable rime.

La quatrième strophe décrit donc un imaginaire: le $t u$ allocutif y est montré phantasmant sa vie et poursuivant sa gloire comme une étoile filante (v. 32). Dans la mesure où cette phantasmatique conduisit Alys Robi à la folie, on voit en quoi cela a pu intéresser Plamondon et Dufresne: leur univers, en effet, est avant tout celui de l'imaginaire socialement brimé (Le parc Belmont, Dioxine de Carbone, etc.). Le texte reconduit ici un cliché, qui est le rêve de gloire hollywoodien. Mais ce cliché n'est pas maladresse, il est citation à peine transposée (et volontairement « cheap ") d'une certaine mythologie américaine.

Dans le texte qui nous occupe, le phantasme de la starification (v. 29-32) donne lieu à quelques-uns des principaux topics de Plamondon tels que les a décrits Jacques Godbout ${ }^{12}$ :

Hôtels de luxe, avion privé

Et lumières de Broadway (v. 33-34)

À cette courte énumération qui ouvre la cinquième strophe, succède une séquence syntaxiquement autonome qui vient clore la seconde partie du texte. Il s'agit des cinq vers (v. 35-39) où sont signalés, d'une part, le nouvel intertexte, Alice au pays des merveilles, et d'autre part, la fin du rêve de starification ( $\propto$ Un beau jour, tout a craquén - v. 39). L'allusion à Lewis Carroll est limpide: Alice/Alys entreprend une traversée du miroir, qui est une traversée des apparences (le spectacle, le cinéma le cinémascope). Le miroir n'est nommé (v. 37) que pour être brisé. La fragilité du miroir appelle l'impact destructeur

12 Jacques Godbout, Plamondon. Un coour de rocker, coll. « Paroles d'ici", Montréal, Éd. de l'Homme, 1988. 
comme la cible appelle la flèche. En même temps que craque le miroir, craque le monde reflété par celui-ci; et craque aussi le sujet qui s'y contemplait (un beau jour, tu as craqué).

Toute la troisième partie du texte - qui va de la sixième strophe à la dernière - constitue une expansion de ce * tout a craqué/tu as craqué». À la lettre, cette troisième partie pourrait être intitulée: mort et résurrection d'une chanteuse populaire.

Le début de la sixième strophe reprend avec de légères mais significatives variantes le texte de la quatrième: par exemple, «tu voyais ta vie» (v. 25) devient « tu vivais ta vie * (v. 40). La gradation est sensible, puisqu'il ne s'agit plus de voir, mais de vivre sa vie comme un film en cinémascope. Cette accentuation du côté irréaliste de la vie de star prépare évidemment la chute, brutale, dans le réel. Dans ce contexte, les «flops» (v. 44) et les « années noires * (v. 45) prennent un singulier relief. Ce relief ne provient que du " contexte du texte", si on peut dire, car les faits narrés restent flous: on compte bien peu de biographèmes, de sorte que le récit demeure allusif. On assiste même à une (relative) banalisation de l'épisode raconté: «Comme n'importe qui » (v. 44), Alys Robi a vécu des hauts et des bas. Cette banalisation a une fonction précise: elle prépare la superposition de la figure de Diane Dufresne à celle d'Alys Robi, qui surviendra à la huitième strophe (v. 56-57) - le conditionnel permettant à Dufresne, on l'a vu, d'imaginer les pensées d'Alys Robi, c'est-à-dire d'y projeter les siennes.

À la différence des strophes qui la précèdent, la septième strophe est rédigée au présent plutôt qu'à l'imparfait. Le passage du temps verbal de la durativité dans le passé (imparfait) à celui du momentané dans l'actuel (présent) est certes notable, encore que le contenu de la strophe tende à multiplier les sèmes indexant la /nostalgie/ - ce qui contribue à rapatrier le passé dans le présent. Il semble d'ailleurs que cette strophe ait pour fonction d'assurer la continuité entre passé et présent. Nous en voulons pour preuve la réitération des motifs de l'«avion privé » et de «Broadway» aux vers 52 et 53 . Mais cette continuité est obtenue au prix de l'inexactitude biographique, car en 1979, il y a belle lurette qu'Alys Robi ne chante plus à Broadway. Cette continuité est, à proprement parler, provoquée. Inexacte sur le plan biographique, elle répond à des exigences thématiques. Elle satisfait également à des 
exigences formelles: c'est elle qui assure le passage à la coda (neuvième strophe), où domine aussi le présent.

L'allusion à Somewhere over the rainbow, la célèbre chanson interprétée par Judy Garland dans Le magicien d'Oz (1939), vient étayer le réseau thématique du texte ${ }^{13}$. Le personnage de Dorothy représente, en effet, une nouvelle Alice, celle-là purement cinématographique. On observe ici un parallèle formel. Mais le parallèle est aussi thématique: somewhere over the rainbow, c'est l'autre côté des choses, l'autre côté du miroir. Ce pays magique, c'est celui d'avant la chute; c'est le monde de l'innocence ${ }^{14}$ - suprême forme d'irréalité.

La huitième strophe est, à plusieurs titres, celle qui justifie l'existence même de la chanson. On sait que Diane Dufresne n'a jamais chanté autre chose que ce qui contribuait directement à dessiner son personnage. Or c'est justement dans cette strophe qu'elle s'approprie l'histoire d'Alys Robi. Outre l'utilisation du conditionnel (v. 55-57), qui favorise cette appropriation, notons celle de l'infinitif chanter, dans «Mais chanter / C'est ta façon / De rester vivante * (v. 58-60). Ces trois courts vers se détachent aisément de l'ensemble: inaugurés par une conjonction d'opposition et par un verbe à l'infinitif, ils forment un bloc repérable. D'où leur importance. L'infinitif chanter, qui est un verbe sans sujet et, plus précisément, un nom d'action, donne à ces vers une portée générale. Il décrit une action intransitive susceptible d'être accomplie par n'importe quel sujet - y compris Diane Dufresne. Il traduit la pure idée marquée par le verbe: le chant, qui part de soi et qui monte, peu à peu. * Chanter c'est une façon de rester vivante * : tel pourrait être, en somme, le crédo de celles qui valorisent dans l'absolu l'acte de chanter. Pour Alys Robi comme pour Diane Dufresne, il semble que chanter soit l'acte premier, le geste décisif.

La dernière strophe constitue la coda proprement dite: $y$ sont reprises quelques formulations déjà exploitées dans ce qui précède. Les vers: "Quand on voit sa vie / Comme un film

13 II est à noter que Judy Garland est aussi connue pour avoir souffert de troubles émotionnels $-\infty$ qui renforce encore le parallele que l'on tente d'établir ici. Rappelons également que Diane Dufresne avait coutume, dans ses spectacles, d'enchainer au vers 54 Somewhere over the rainbow, puis de reprendre Alys en cinémascope.

14 Pour avoir une idée de la place de l'innocence dans l'univers d'Alys Robi, il n'y a qu'à consulter sa pseudo-autobiographie, qui n'est qu'un récit nostalgique des années d'innocence qui ont précédé la période d'internement. 
en cinémascope ", y sont repris trois fois, avec ou sans modification. Les " hits " et les « flops » du vers 44 y sont également évoqués. Dans cette strophe, on lit en raccourci le parcours entier de la chanson: "top" rime ici, pour la seule et unique fois du texte, avec "stop" (v. 66 et 68), qui le contient, l'embrasse, comme si «top* était contenu dans «stop»: métaphore de la courbe à son apogée, qui programme déjà son propre déclin, d'ailleurs inscrit dans le tracé même de sa trajectoire.

L'analyse de la dernière strophe d'Alys en cinémascope met au jour, de façon exemplaire, les deux registres qui se partagent l'ensemble du texte: un registre narratif et un registre métaphorique. Ainsi, à un synopsis biographique plutôt mince, mais néanmoins insistant, se greffe un tissu métaphorique proliférant (la star, le cinéma, Alice, etc.) qui enrichit le texte, mais qui contribue également à le «brouiller». Dialectique, le texte enchevêtre le référentiel et le symbolique; c'est pourquoi il reste particulièrement allusif.

Rappelons en terminant que, pour aborder Alys en cinémascope, certaines connaissances biographiques sont requises. On ne peut bien comprendre le texte sans connaitre d'abord l'histoire d'Alys Robi; on doit également connaître celle de Diane Dufresne; enfin, il est préférable d'être familier avec l'univers de Luc Plamondon. Cependant, il nous est apparu qu'une démarche attentive à la composante métaphorique du texte était plus rentable qu'une démarche simplement biographique ou psychanalytique. C'est ce qui, nous l'espérons, explique et justifie notre parti pris d'analyse.

Alys en cinémascope nous paraît exemplairement lisible à travers le prisme (la métaphore) du cinéma. Les effets de ce prisme, on l'a vu, se manifestent sur tous les plans. Le cinéma est, si on peut dire, la matrice du texte; il est son générateur formel et thématique. L'auteur a fort bien choisi son prisme: que demander de mieux, en effet, que d'apprendre l'histoire d'Alys - qui se fait son cinéma - par l'entremise d'un regard qui lui-même emprunte les tours du langage cinématographique ${ }^{15}$ ? Ne dirait-on pas qu'ici la représentation et la transposition, effets spectaculaires et spéculaires, règnent en maîtres?

15 Ainsi donc, on peut voir un champ-contrechamp dans le contraste entre la sixième strophe et la septième. Et que dire de la syntaxe de la première strophe, qui anticipe celle des vidéos-clips? 